\title{
Review of Thermodynamics of Systems that Embrace Transfer of Electric and Magnetic Energies
}

\author{
Salama Abdelhady \\ Department of Mechanical Engineering, Faculty of Energy Engineering, Aswan University, Aswan 81528, Egypt
}

\begin{abstract}
Thermodynamics is a branch of physics which deals with the energy flow into systems. There are two principal laws of thermodynamics which are described on separate slides. The first law of thermodynamics relates to the transfer of various forms of energy, as heat, electric, magnetic and chemical energies to produce mechanical work. This law is sometimes taken as the definition of internal energy, and introduces an additional state variable, enthalpy. The first law of thermodynamics allows for many possible states of a system to exist. But experience indicates that there is only one direction for natural processes to proceed. This leads to the second law of thermodynamics and the definition of another state variable called entropy. The second law stipulates that the total entropy of a system plus its environment cannot decrease; it can remain constant for a reversible process but must always increase for irreversible or natural processes. However, the limited number of authors is concerned by systems that involve the transfer of electric and magnetic energies in addition to the flow of heat in studying a general thermodynamic system. The submitted review follows an entropy approach that introduces clear definitions of the electric charge and magnetic flux to clarify ambiguities of the natures of such fluxes in literature. So, the first and second laws of thermodynamics are applied on thermo-electromagnetic processes that embrace the flow of mechanical, thermal, electric, and magnetic energies into systems that involve fluids or solids. Accordingly, it was possible to modify redundancies in the SI system of units. Following the entropy approach, it was possible to cast the Maxwell's equations into an energy frame of reference to explain the discovered Tesla's wireless power transmission as "Electrical Radiant Energy", and the MIT discovery of wireless power transmission of the magnetic flux as "Evanescent Waves" and to clarify fuzziness of the duality confusion by specifying a unique property for the electron as a particle and a unique property for the light as a wave. Consequently, it was possible also to prove that the semiconductors efficiency responds mainly to the concentration of the incident solar energy, i.e. incident solar energy per unit area, and it is partially influenced by the wavelength.
\end{abstract}

Key words: Thermodynamics, electromagnetism, potentials energy, entropy, units.

\section{Introduction}

Most of literatures which deal with thermodynamic systems are concerned mainly by transfer of mechanical and thermal energies [1]. Similarly, most of literatures which deal with flow of electromagnetic energy are concerned mainly by solids that have electric or magnetic properties [2]. Both analyses are ineffective when dealing with a general thermodynamic system that may involve fluids and it is subjected to the transfer of heat, electric, magnetic and mechanical energies [3]. Reviewing literature that deals with the transfer of electric and magnetic energies; most of them are concerned mainly by

Corresponding author: Salama Abdelhady, Ph.D., professor, research fields: energy systems. momentum conservation while they gave a narrow space to the energy and entropy changes which are the main concern in energy analysis [4]. Such limitations may be due to the absence of clear definitions of the natures of the electric charge and magnetic flux as forms of energy in transfer [5]. So, we cannot find a clear energy analysis that plausibly explain the transfer of electric and magnetic energies in space as the discovered Tesla's wireless power transmission [6] and the wireless power transmission by magnetic resonance [7]. The duality confusion represents also a riddle in the energy analysis of electromagnetic systems [8]. While Maxwell's equations of electromagnetic waves express mainly the flow of energy in space, it has not yet been casted into an Energy frame of reference that depends on energy 
parameters [9]. Additionally there are found redundancies in SI system due to inserting ambiguous units, as Henry, Tesla and Ampere, which represents units of variables that should be related directly to the energy units [10]. Similarly; the definition of the energy quanta, as postulated by Planck, has not any thermodynamic foundations [11]. Similarly, there is still an obstacle in finding a plausible explanation of the broken limit of the efficiency of the photovoltaic cells as defined by Shockley and Queisser [12].

So, it will be reviewed in this study an entropy approach to advanced analysis of thermodynamic systems that embraces the transfer of electric and magnetic energies to solve the previously discussed ambiguities found in literature. Such review starts by discussing the similarity of the natures of the electric charge, magnetic flux and the heat energy in Section 2.

Then Section 3 and Section 5 will review the advanced applications of the first and second laws of thermodynamics on a system that embraces the transfer of electric and magnetic energies. Section 4 will be devoted to solve redundancies in SI system through the tracked entropy approach. It will be reviewed in Section 6 how casting the Maxwell's equations of electromagnetic waves into an energy frame of reference that replaces the time by entropy may lay to find robust definitions of the natures of electric charge and magnetic flux and to find plausible explanations of the discovered Tesla's wireless power transmission as "Electrical Radiant Energy" and the MIT discovery of wireless power transmission of the magnetic flux as "Evanescent Waves". Consequently, it will lead to define the quantization of energy and to modify the Einstein's description of the photovoltaic effect and the Shockley and Queisser efficiency limit of the efficiency of photovoltaic cells. Section 7 will be concerned by reviewing the definitions of thermodynamic potentials in the field of thermo-electromagnetic systems.

\section{Similarity and Reversible Interchangeability of Heat, Electric Current and Magnetic Flux}

Investigating the definitions of the thermoelectric effect in literature, it can be understood that heat and electric current have the same nature and that the conversion of heat into electric current, and vice versa, can be described as a process of replacing the thermal potential of heat by an electric potential or vice versa [11]. Such conclusions can be deducted by reviewing the discoveries of Joule, Seebeck, Peltier, and Thomson [12]. According to Joule's law, the flow of an electric current into a conductor generates heat that is dissipated to surroundings. This proves the common nature of flow of electric charge and of heat as energy in transfer [13]. The German physicist Thomas J. Seebeck discovered the thermocouple which generates an electromotive force when its junctions are at different temperatures [14]. Accordingly, the Seebeck effect was utilized in thermoelectric generators where heat is converted into electric current when the thermal potential between the junctions of the thermocouples is converted into electric potential or electromotive force [15]. In 1834, the French physicist Jean C. A. Peltier discovered an effect inverse to the Seebeck effect where he passed an electric current through a thermocouple, then the temperature of one junction increases and the temperature of the other decreases. So, the Peltier effect was considered also as a proof of the reversibility of conversion of electric current into heat by replacing the electric potential of the electrical current by thermal potential [16]. The Scottish scientist William Thomson (later Lord Kelvin) discovered in 1854 that if a temperature difference exists between any two points of a current-carrying conductor, heat is either evolved or absorbed depending upon the material [17]. It had been shown also that the Seebeck effect is a result of the combined Peltier and Thomson effects assuring the similarity of the natures of heat and electric current and their reversible 
interchangeability by replacing their potentials through the discovered effects [18].

Reviewing Faraday's experiment of magnetic induction, Fig. 1, it is possible to explain its results as follows: the electric current in the primary coil is converted by induction into magnetic flux in the core; then the induced magnetic flux of the core is converted once more into an electric current in the secondary coil [19]. Such way of understanding or description proves that both fluxes, electric and magnetic, should have similar natures and that their conversion process is experienced by exchanging their potentials, electric or magnetic, in the primary and secondary coils [20].

Reviewing the results of a simple experiment, Fig. 2, that used a permanent magnet immersed in a water basin to attract iron balls along an inclined smooth glass plane; the heat lost from the water was found equivalent to the done magnetic work in attraction of the balls along the shown plate [21]. Such results may be considered as a proof that magnetic work used to work used to attract the balls is equally

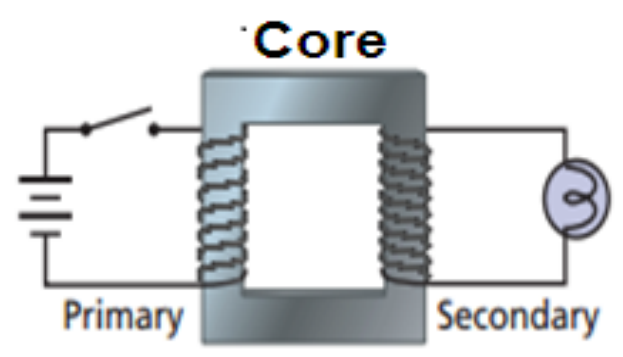

Fig. 1 Faraday's experiment of magnetic induction.

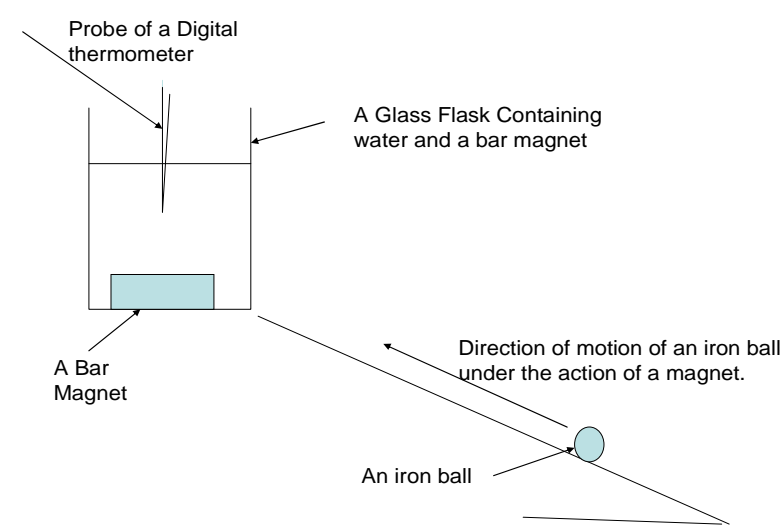

Fig. 2 An experiment that shows the calorific value of magnetism. substituted by the heat lost from the water; or that potential of heat is replaced by magnetic potential to substitute the lost magnetic energy from the magnet. Similarly, it was possible to measure the equivalence of the heating rates, in magnetic fluids, to the subjected alternating magnetic power [22]. Reviewing also the definition of the magnetocaloric effect in literature as applied in refrigeration; it is possible to deduce the same conclusion of similarity of the natures of heat and magnetic flux [23]. Consequently, it is possible to postulate the similarity of the natures of the magnetic flux and heat and their reversible interchangeability through exchanging their potentials [21].

Finally, heat, electric and magnetic energies were concluded as forms of energies of similar and of equivalent natures and that they are reversibly interchangeable by exchanging their potentials through reversible phenomena as thermoelectric effect, magnetocaloric effect, and induction [24].

According to quantum literatures, it is stated that the light, as a spectrum of thermal radiation, the electric charge and magnetic flux are quantized into discrete quantities [25]. Such common property of discretization can be considered also as additional proof of the similarity of the natures of these energies.

\section{The First Law of Thermodynamics of Electromagnetic System}

Studying a thermodynamic system which is subjected to flow or transfer of heat, electric current and magnetic flux while it is performing a mechanical work; the energy conservation principle, or the first law of thermodynamics, is expressed as follows [26]:

$$
d U=\delta Q_{t h}+\delta Q_{e l}+\delta Q_{m a g}-P d V
$$

According to references in electrodynamics, Eq. (1) is expressed as follows [27]:

$$
d U=T d S+E d+H d M-P d V
$$

In Eq. (2), $T, E$, and $H$ are the thermal, electric, magnetic potentials of heat, electric current and magnetic flux, $\mathrm{S}$ is the entropy, is the electric 
polarization, $M$ is the magnetization, $P$ is the pressure, and $V$ is the volume.

Comparing the energy Eqs. (1) and (2), the following equalities can be concluded [28]:

$$
\begin{gathered}
\delta Q_{t h}=T d S \\
\delta Q_{e l}=E d \\
Q_{m a g}=H d M
\end{gathered}
$$

According to statistical thermodynamics, it was found the following relation between the change of entropy $\Delta S$ and polarization [29]:

$$
\Delta S=\frac{1}{2} \beta^{2}
$$

where $\beta$ represent a temperature-independent phenomenological coefficient [29]

Eq. (6) indicates the dependence of polarization on entropy. So, it is possible to rewrite Eq. (6) in terms of the thermodynamic property " $S$ " as follows:

$$
\delta Q_{e l}=E d f_{e}(S)
$$

According to the proved reversible interchangeability and similarity or equivalence of the flow of heat and electric charges; it is reasonable to express the differential of the charge flow in " $\delta Q_{e l}$ " in Eq. (7) directly in directly terms an entropy differential $d S$ instead: $d f_{e}(S)^{\prime}$ ' in analogy to Eq. (3) for the flow of thermal energy as both are similar [27]: i.e.:

$$
\delta Q_{e l}=E d S
$$

It has been found also a relation between magnetization and entropy of a magnetic system as follows [30]:

$$
S=k_{B} \ln (M+1)
$$

Such relation proves also the mutual dependence between entropy and magnetization. Consequently, it is possible to replace the differential " $d M$ " in Eq. (9) by a differential of a function in terms of the thermodynamic property " $S$ " as follows:

$$
\delta Q_{\text {mag }}=H d f_{m}(S)
$$

According to the reviewed similarity of heat, flow of electric charge and flow of magnetic flux, it is reasonable to replace the differential: " $d f_{m}(S)$ " by the entropy differential $d S$ in analogy to Eq. (3) for the flow of thermal energy as both are similar [27]:

$$
\delta Q_{\text {mag }}=H d S
$$

So, the first law of thermodynamics that embraces the flow of heat, electric charge and magnetic flux can be casted as follows [27]:

$$
d U=T d S+E d S+H d S-P d V
$$

Eqs. (8) and (11) express the flows of electric and magnetic energies as a potential force times a differential of the corresponding increase entropy of the system and their integrals can be expressed in property diagrams between the entropy and the electric or magnetic potentials of the system. The differential of entropy is an exact differential as the entropy is a point function or a property of the system. Accordingly, Eq. (12) can be considered as a fundamental equation as it involves only properties of the system [27].

However, traditional literature of electromagnetism stated the energy equation that embraces the transfer of electric and magnetic energies is as follows [30]:

$$
d U=T d S+E \delta D+H \delta B-P d V
$$

According to such literature, $D$ is the displacement current and $B$ is the magnetic flux. However, the differentials $\delta D$ and $\delta B$, in Eq. (13) express differentials of energy in transfer which are not differentials of properties of the considered thermodynamic system; so both are non-exact differentials and their integrals $\int E \delta D \& \int H \delta B$ cannot be represented in a property diagram. Additionally, the directional current has the dimensions of energy flow rate and the product of its multiplication by the electric potential " $E$ ", i.e. the term " $E \delta D$ ", will not have the dimensions of energy as the other terms of the equation. Similarly, the magnetic flux " $B$ " have the dimensions of energy, i.e. the term " $H \delta B$ " will not have the dimensions of energy as the other terms in such energy equation. On the other hand, the differential $d \mathrm{~S}$ in the terms of thermal, electric and magnetic energies in Eq. (12) is an exact differential as it is a differential of a property and the integrals " $\int T d S$ ", " $\int E d S$ " and " $\int H d S$ " 
represent the flows of thermal, electric and magnetic energies can be represented into property diagrams [24]. So, Eq. (12) can be considered as a fundamental energy equation as all of its terms are properties of the system and all its differentials are exact ones while Eq. (13) cannot be considered as fundamental equation as the differentials of the displacement current " $D$ " and the magnetic flux " $B$ " are non-exact and depends on the path [26].

\section{Units and Dimensions Electromagnetism}

The electric potential "E" is measured in volt. Similarly, the thermal potential is measured by thermocouples in volt [31]. During their experimental work to compare the performance of some common thermocouples; Kumar and others recorded variations of the generated EMF (Electromotive Force), in volts, from an Aluminum-Iron thermocouple by the influence of the magnetic potential similar to its influence by the temperature difference [32]. According to such results and to the proved similarity and reversible interchangeability of thermal, electric and magnetic energies, it is possible to state the volt as a common unit for the measurement of the electric, thermal and magnetic potentials [33].

So, the entropy Eqs. (3), (8) and (11) will be of the same unit in Joule/volt.

Dividing both sides of Eq. (3) by the time differential " $d t$ ", it is possible to estimate the rate of heat flow as follows [28]:

$$
\dot{Q}_{\text {thermal }}=T \dot{S}
$$

Similarly, dividing both sides of Eq. (8) by the time differential " $d t$ ", the electric power as a rate of electric energy flow can be expressed also as follows [28]:

$$
\dot{Q}_{\text {elec }}=E \dot{S}
$$

Similarly, the rate of flow magnetic energy can be expressed according to Eq. (11) as follows [28]:

$$
\dot{Q}_{\text {mag }}=H \dot{S}
$$

According to literature of electromagnetism, the electric power is estimated according to the following Eq. [34]:

$$
\dot{Q}_{\text {elec }}=E I
$$

" $\Gamma$ " in Eq. (18) is defined as the electric current or as the rate of flow of electric charge. As the electric charge is a form of energy, the rate of flow of electric charge, or " $T$ ", represents actually the rate of flow of energy or the electric power and this contradicts its multiplication by the electric potential " $E$ " in Eq. (18) to determine the electric power. Such contradiction leads to confusions in the SI system of units and is found as a source of error in the homogeneity of dimensions of Ampere's law [33]. If the product of $E * I$ is the electric power, so the current "I" should be defined as the rate of flow of electric charge per unit drop in potential, i.e. W/Volt. Comparing the terms on the R.H.S of Eqs. (16) and (18), it is possible to define the term "I" as the rate of flow of entropy " $\dot{S}$ " that is expressed by the following equation [33]

$$
I=\dot{S}
$$

In other words, the Ammeter measures the rate of entropy flow and does not measure a rate of charge flow.

According to the proved interchangeability of electric and magnetic energies, the magnetic flux or the rate of flow magnetic energy should have also the same dimensions or units as the electric current. It is defined in literature by the following equation [34]:

$$
\dot{Q}_{m a g}=H B
$$

When comparing Eq. (11) to Eq. (17), it can be found that the magnetic flux " $B$ " is indicating also the rate of entropy flow associated by the flow of magnetic energy [33]; i.e.:

$$
B=\dot{S}
$$

Such approach leads to introduce a Universal System of Units, as summarized in Table 1 [33]. Such system defined the unit of electric current "I" and the magnetic flux " $B$ " in $\mathrm{W} /$ Volt and assigned the same unit to the thermal, electric and magnetic potentials in Volt and the same unit for their fluxes in Joule/Volt [33]. This system succeeds in deleting the confusions 
in the SI system of units between similar and analogous flows of the thermal, electric and magnetic energies as found in Table 2 [35].

As the increase of entropy by heat, electric and magnetic energies has the same unit, in Joule/Volt, the total increase of a system that embraces the transfer of such energies was estimated directly as a sum of increase of entropies resulted such flows according to the following equation [24]:

$$
S_{2}-S_{1}=\int_{1}^{2}\left[d S_{t h}+d S_{\text {elect }}+d S_{\text {mag }}\right]
$$

\section{Second Law of Thermodynamics of Electromagnetic System}

Generally, flows in thermodynamic systems are driven by forces. Accordingly, flows and forces occur in conjugate pairs in thermodynamics of a general electromagnetic system. This means the heat flow is driven by differences in temperature between the system and the surrounding, volume flow is by differences in pressure, electric charge flow by differences in electrical potential, the magnetic flux by difference in magnetic potential, and mass flow by differences in concentration [36].

The second law of thermodynamics provides a rule that describes the direction of change in a system in the absence of external forces. It depends on natural evidence that heat flows from warm objects to cold objects, that objects fall downward in a gravity field, that electric current flows from high potential to low potential, the magnetic flux emerges from magnets of high magnetic potential to iron rods of zero or less magnetic potential and solutes diffuse from regions of high concentration into regions of low concentration [37]. So, the second law is a rule which captures these facts in a remarkably concise way. Essential to the second law of thermodynamics is the idea of a reversible flow. A flow is reversible when it is driven by an infinitesimal force, i.e. a difference in the force which is so close to zero that a small change in the magnitude of the force at the appropriate place can reverse the direction of the flow [38]. The total change in entropy (system plus surroundings) during a reversible heat transfer process which is defined as transferring the same heat to surrounding through infinitesimally small difference is equal to zero according to the following equality [39].

$$
\text { i.e. } \oint \frac{\delta Q_{t h}}{T}=0
$$

According to laws of physics, as Eq. (23) indicates the cycle integration of the differential " $\frac{\delta Q_{t h}}{T}$ " is zero, then such differential is a differential of property, named according to the second law as entropy [39]. In real processes where the temperature of the system should be higher than the temperature of surroundings; the total entropy change of the system and the surroundings exceeds the zero value as follows [40]:

$$
\oint \frac{\delta Q_{t h}}{T} \geq 0
$$

So, the second law of thermodynamics introduced also the entropy as a property of thermodynamics that measures the irreversibility of processes in thermodynamic systems. Entropy of a system is traditionally defined in terms of its statistical concept according to Ludwig Boltzmann as follows [41]:

$$
S=k_{B} \cdot \ln \Omega
$$

$k_{B}$ : Boltzmann's constant $=1.38 \times 10^{-23}, \Omega$ is the number of arranging the microstates of a system at specified total internal energy. According to Boltzmann definition of entropy and to the concepts of the second law of thermodynamics, the entropy was proven to indicate also the arrow of time from the statistical behavior of a large number of molecules obeying the conservation of momentum and energy [42].

Similar to the irreversible heat transfer through a substantial temperature difference, the electric energy is transferred also through a substantial electric potential and the magnetic flux is transferred also through a substantial magnetic potential. In other words, the transfer of electric current or magnetic flux 
may have similar irreversibilities as the transfer of heat. However, literature ignores such irreversibilities which represent as similar sources of irreversibilities as the heat transfer. Applying the second law of thermodynamics to systems that embrace the transfer of electric current and magnetic flux; the irreversibilities of such transfer was expressed by the following equation [24]:

$$
\begin{aligned}
\oint \frac{\delta Q_{e l}}{E} & \geq 0 \\
\oint \frac{\delta Q_{m a g}}{H} & \geq 0
\end{aligned}
$$

Eqs. (26) and (27) are similar to Eq. (21) for heat transfer that expresses the natural flow of heat through a substantial temperature difference. However, transferring the electric and magnetic fluxes through infinitesimally small differences, the following equalities will be valid [28]:

$$
\begin{gathered}
\oint \frac{\delta Q_{e l}}{E}=0 \\
\oint \frac{\delta Q_{m a g}}{H}=0
\end{gathered}
$$

Eq. (23) was considered as an approach to define the entropy as a property in thermodynamics. Accordingly; Eqs. (28) and (29) prove also the entropy as a property in electromagnetism systems which defined by the following equations [28].

$$
\begin{gathered}
d S=\frac{\delta Q_{e l}}{E} \\
d S=\frac{\delta Q_{m a g}}{H}
\end{gathered}
$$

Consequentially, the second law of thermodynamics of electromagnetic systems introduced the entropy as a property of the system and as a measure of the irreversibility of the processes of transfer of electric and magnetic energies. So, the flow of electric and magnetic energies was represented in an E-s property diagram and an $\mathrm{H}$-s property diagrams as shown in Figs. 2 and 3, similar to representation of heat in a T-s diagram, Fig. 1 [28].

\section{Definitions of the Nature of Heat, Electric Charge, Magnetic Flux and the Energy Quanta}

The nature of flow of heat is identified as flow of electromagnetic waves. Maxwell had succeeded in describing the flow of electromagnetic waves by simultaneous flow of electric and magnetic energies according to the following mathematical equations [43].

$$
\begin{aligned}
& \left(\nabla^{2}-\frac{1}{c^{2}} \frac{\partial^{2}}{t^{2}}\right) \cdot \mathrm{E}=0 \\
& \left(\nabla^{2}-\frac{1}{c^{2}} \frac{\partial^{2}}{t^{2}}\right) \cdot \mathrm{H}=0
\end{aligned}
$$

where $c$ is the speed of light in the medium, in vacuum $c=c_{0}=299,792,458 \mathrm{~m} / \mathrm{s}$. Replacing the time in Maxwell's equation by entropy, the Maxwell's equations will be in an energy frame of reference formed of the electric and magnetic fields, "E" and "H", and entropy "S" as follows [44]:

$$
\begin{aligned}
& \left(\nabla^{2}-\frac{1}{c^{2}} \frac{\partial^{2}}{s^{2}}\right) \cdot \mathrm{E}=0 \\
& \left(\nabla^{2}-\frac{1}{c^{2}} \frac{\partial^{2}}{s^{2}}\right) \cdot H=0
\end{aligned}
$$

Fig. 4 represents such modified Maxwell's equations in an energy frame of reference where the swept areas by the electromagnetic wave in the E-s and

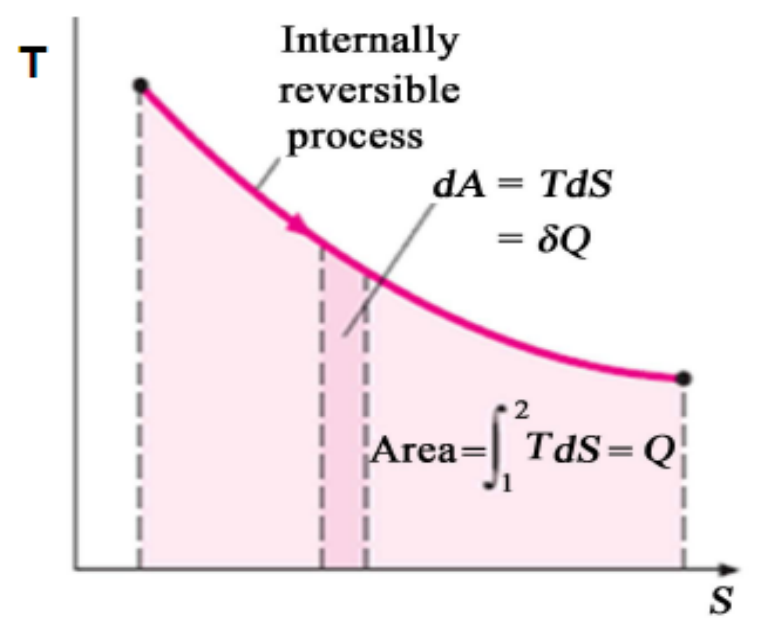

Fig. 3 Graphical representation of heat transferred reversibly to a system in a T-s property diagram. 


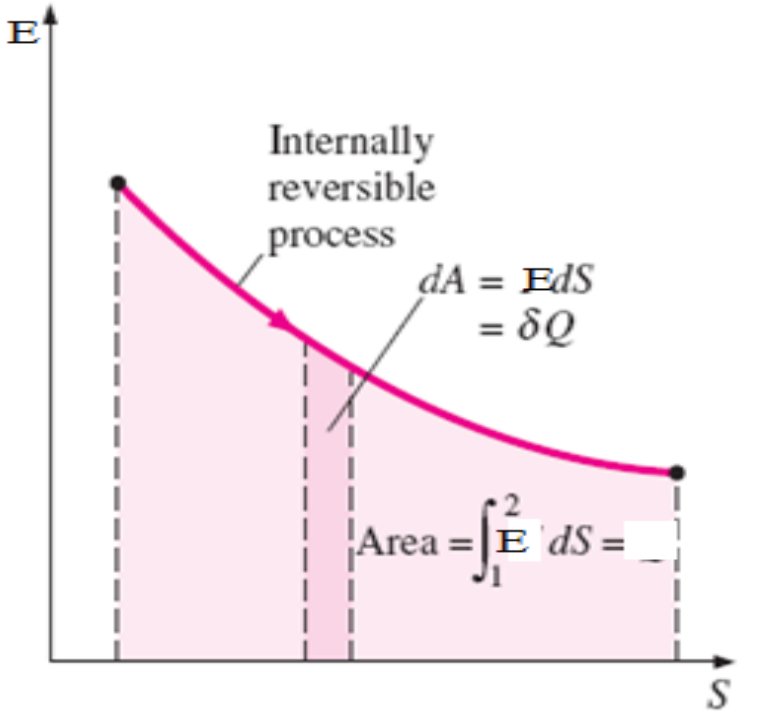

Fig. 4 Graphical representation of electric energy transferred reversibly to a system in an E-s property diagram.

$\mathrm{H}-\mathrm{s}$ planes represent the flowing electric and magnetic energies [28]. However, the replacement of the time in Maxwell's equation by entropy fits also Eddington's conclusion which found the time's arrow is a property of entropy alone as the entropy is an energy property that measures of randomness increase in time [45]

If we denote the swept area per an electromagnetic cycle, or imparted energy per wave-pulse, by the symbol " $h$ " in Joule/cycle, them this energy pulse can be estimated as the sum of the energies imparted by the electric wave and by the magnetic wave in one pulse, or $1 / v$ second, as follows [46]:

$$
\mathrm{h}=\int_{0}^{2 \pi}\left|E d S_{\text {elect }}\right|+\int_{0}^{2 \pi}\left|H d S_{\text {mag }}\right|
$$

Accordingly, it is possible to calculate the energy flow per unit time in an electromagnetic wave of frequency " $v$ " cycle/s, or $\mathrm{Hz}$, as follows:

$$
\dot{W}=\mathrm{h} v \text { Watt / wave of frequency " } v \text { ". }
$$

According to equation (37), the value of $\dot{W}$, or $\mathrm{h} v$, represents the rate of flow of energy imparted by electromagnetic wave of frequency " $v$ " per unit time.

In his scientific work to explain the photovoltaic effect, Einstein postulated that light was made of packets of energy, or energy quanta called as "photons," where each photon carries a specific energy related to its wavelength as follows:

$$
e_{\text {photon }}=h v \text { Joule }
$$

" $h$ " is called Planck's constant. Planck estimated the magnitude of such quanta for a wave whose frequency

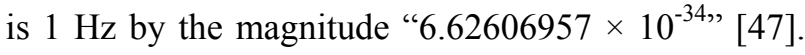
Substituting $v=1$ in Eq. (38), we get the following results:

$$
\begin{gathered}
e_{\text {photon, } v=1}=h= \\
6.62606957 \times 10^{-34} \text { Joule/cycle }
\end{gathered}
$$

According to Eq. (39), Planck's constant defines actually the magnitude of energy pulse, denoted previously as " $h$ ". So, it is possible to state the following equality:

$$
h \equiv \mathrm{h} \quad \text { Joule/energy-pulse }
$$

Accordingly, the quantity " $\mathrm{h} v$ " or " $\mathrm{h} v$ " expresses the rate of flow of energy of an electromagnetic wave whose frequency is " $v$ " in Watt/wave [24].

As an example to realize the results of the introduced definition of Planck's constant, we may consider the green light at frequency " $v "=5.76 \times 10^{14}$ cycle/sec. Then, the rate of energy flow per wave of the green light can be estimated as follows:

$$
\begin{gathered}
\dot{W}=\mathrm{h} v=\left(6.626 \times 10^{-34} \mathrm{~J}\right) \cdot\left(5.76 \times 10^{14} \mathrm{~s}^{-1}\right) \\
=3.82 \times 10^{-19} \text { Watt } / \text { wave of green light }
\end{gathered}
$$

The value of the solar constant, according to literature, is equal to $1,366.1 \mathrm{~W} / \mathrm{m}^{2}$. Hence, we may calculate the number of waves in a bundle imparted on $1 \mathrm{~m}^{2}$ of the earth as follows:

$$
\begin{gathered}
\text { per sq.meter }=\frac{1366.1}{3.82 \times 10^{-} 19} \frac{\mathrm{Watt} / \mathrm{m}^{2}}{\mathrm{Watt} / \text { wave }}=3.6 \times 10^{21} \\
\text { wave } / \mathrm{m}^{2} .
\end{gathered}
$$

Similarly, it is possible to count the number of wave per glass-fiber of diameter $62.5 \mu \mathrm{m}$ as follows:

$$
\text { per glass fiber }=\frac{\pi d^{2}}{4} \text { sq.meter }=10 \times 10^{10}
$$$$
\text { waves. }
$$

According to the previous conclusions, it is possible to modify Einstein's description of the specified limit of threshold frequency. According to Eq. (41); this limit is not determined by the energy of a quanta but it is determined by the rate of flow of energy of the waves per unit area that is capable of crossing the p-n 
junction of the photovoltaic cell and gains its potential to be converted into an electric current. This truth of this explanation is sustained by the results of using CPV (Concentrating Photovoltaic) where the intensity of solar radiation allows the electromagnetic waves of lower frequencies to be converted into electric current due to increasing the rate of flow of energy per unit area. This represents a plausible explanation for getting a high efficiency of CPV that exceeds the limit determined by Shockley, W. and Queisser, H. J. [48].

According to the discovered Tesla's "Radiant Energy" as normal transfer of electric current in space as waves, it is possible to postulate the nature of electric charge as ionized electromagnetic waves or as electromagnetic waves that have an electric potential [49]. Depending on the introduced coordinates in Maxwell's equations that involves the entropy as energy coordinate, it was possible to presume a graphical representation of the electric current as flow electromagnetic waves whose electric component has an electric potential " $\Delta E$ " as seen in Fig. 5 [28]. This graph interprets the following solution of the modified Maxwell's Eqs. (34) and (35) for a wave flowing with an electric potential " $+/-\Delta E$ " [49]:

$$
\mathrm{E}(\mathrm{r}, \mathrm{s})=g_{1}(\omega \mathrm{s}-\mathrm{kr})+/-\Delta \mathrm{E}
$$

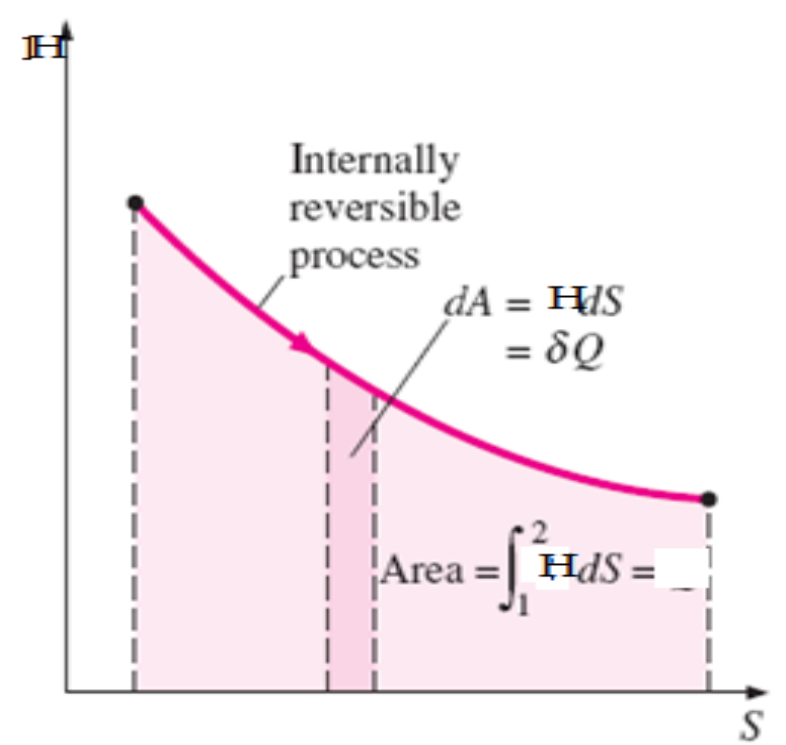

Fig. 5 Graphical representation of magnetic energy transferred reversibly to a system in an $\mathrm{H}$-s property diagram.

$$
\mathrm{H}(\mathrm{r}, \mathrm{s})=g_{2}(\omega \mathrm{s}-\mathrm{kr})
$$

The potential of the electric component of the flowing wave will be " $+\Delta \mathrm{E}$ " for the flow of a positive charge and " $-\Delta \mathrm{E}$ " for a negative charge [28].

According to the found similarity and interchangeability of electric charge and magnetic flux by Faraday's experiment; the nature of the magnetic flux was identified also as a flow of electromagnetic waves that have a magnetic potential [21]. According to such definition, it was possible to find plausible explanation of the MIT discovery of wireless power transmission of the magnetic flux, as "Evanescent waves", by the discovered magnetic resonant coupling [50]. Accordingly, the previously introduced entropy approach defined the nature of magnetic flux as electromagnetic waves whose magnetic component has a magnetic potential as a plausible explanation of the MIT experiment [51]. Such definition is described mathematically by the following equations, as a solution of the modified Maxwell's equations Eqs. (34) and (35), and is represented graphically in Fig. 6 [21]:

$$
\begin{gathered}
\mathrm{E}(\mathrm{r}, \mathrm{s})=g_{1}(\omega \mathrm{s}-\mathrm{kr}) \\
\mathrm{H}(\mathrm{r}, \mathrm{s})=g_{2}(\omega \mathrm{s}-\mathrm{kr})+/-\Delta \mathrm{H}
\end{gathered}
$$

The potential of the magnetic component of the flowing electromagnetic wave defined by Eq. (41) by the term $+/-\Delta \mathrm{H}$ which determines the strength and direction of the magnetic field associated by the flowing

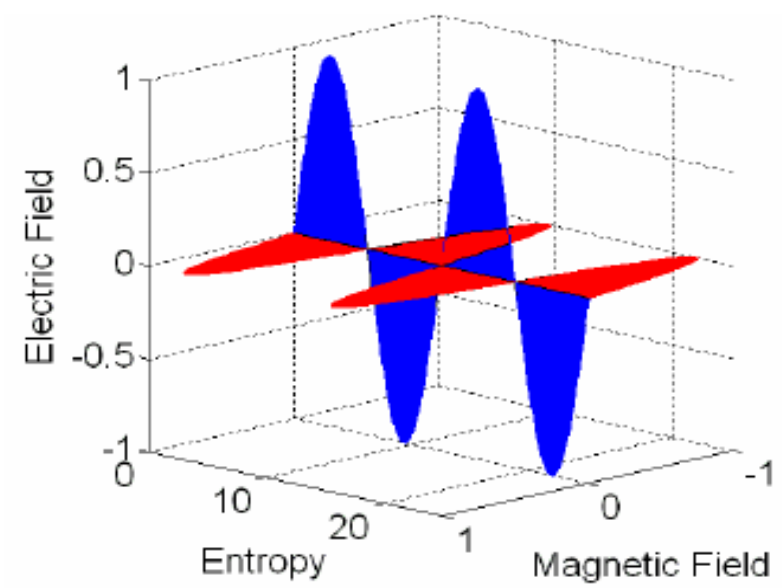

Fig. 6 Flow of electromagnetic waves in an energy frame of reference that shows the electric energy flow in an Electric field-entropy plane and the magnetic energy flow into a Magnetic field-Entropy plane [28]. 
magnetic flux as electromagnetic waves [28].

As a conclusion, the entropy approach succeeded in showing energy pulses, which was wrongly considered as energy quanta in Fig. 3. It was possible also to find a plausible definition of electric charge and magnetic flux by Eqs. (39) and (42) and as shown in Figs. 4 and 5.

\section{Potentials in Thermodynamics of Electromagnetism}

The following equation was stated, according to an entropy approach, as a fundamental equation of thermodynamic of electromagnetic system as all its terms express properties of the system [28].

$$
d U=T d S+E d S+H d S-P d V
$$

Trials have been made to define thermodynamic potentials in electromagnetism [51, 52]. However, without a fundamental energy equation, as Eq. (43), such trials were not successful. As the differentials " $d S$ " in Eq. (43) belong to the same property $S$ and the potentials " $T, E$ and $H$ " have the same unit, it was possible to rearrange the terms in Eq. (43) as follows:

$$
d U=(T+E+H) d S-P d V
$$

According to Eq. (44), the internal energy is a function of the extensive properties $S$ and $V$, i.e. we have:

$$
U=U(S, V)
$$

Accordingly, its differential can be expressed as follows:

$$
d U=(\partial U / \partial S)_{V} d S+(\partial U / \partial V)_{S} d V
$$

Comparing Eq. (44) and (46), it is possible to prove the following equalities [56]:

$$
\begin{gathered}
(\partial U / \partial S)_{V}=T+E+H \\
(\partial U / \partial V)_{S}=-p
\end{gathered}
$$

According to Eqs. (44) and (46), we may consider the following cases [56]:

In case of absence of electric and magnetic fields;

$$
\begin{array}{r}
E=H=0 ;(\partial U / \partial S)_{V}=T, \\
(\partial U / \partial V)_{S}=-p_{\text {mech }}
\end{array}
$$

In case of absence of electric and thermal fields;

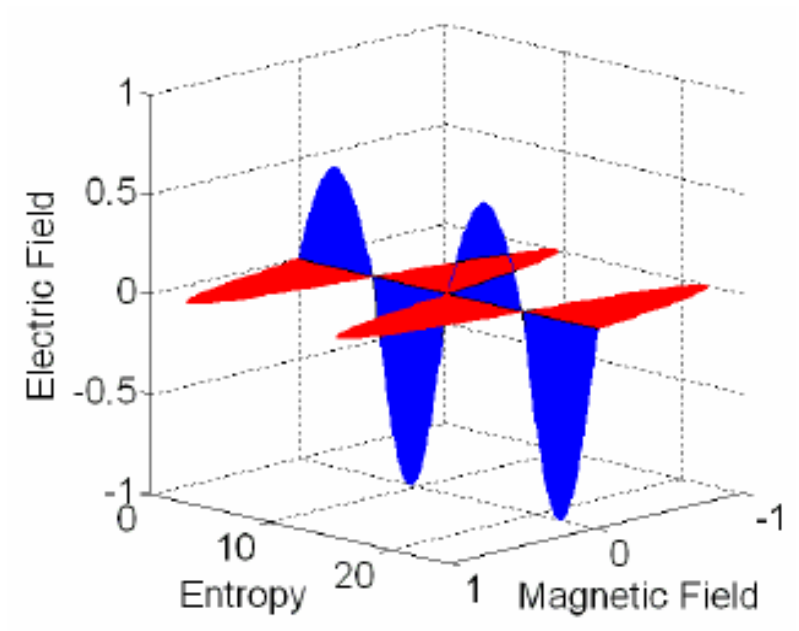

Fig. 7 Flow of electric charges as E. M. waves of non-zero electric potential [52].

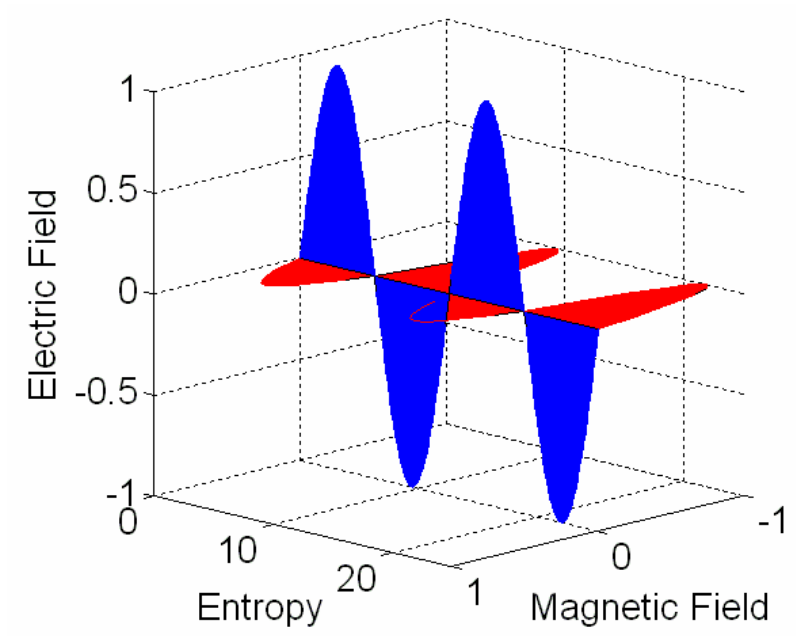

Fig. 8 Graphical representation of magnetic flux [52].

$$
\begin{gathered}
E=T=0 ;(\partial U / \partial S)_{V}=H, \\
(\partial U / \partial V)_{S}=-p_{\text {elect }}
\end{gathered}
$$

In case of absence of magnetic and thermal fields;

$$
\begin{gathered}
T=H=0 ;(\partial U / \partial S)_{V}=E, \\
(\partial U / \partial V)_{S}=-p_{\text {magnetic }}
\end{gathered}
$$

In case of a system influenced simultaneously by thermal and electric fields:

$$
\begin{aligned}
H=0 ;(\partial U / \partial S)_{V} & =T+E,(\partial U / \partial V)_{S}= \\
& -\left(p_{\text {mech }}+p_{\text {elect }}\right)(52)
\end{aligned}
$$

In case of a system influenced simultaneously by thermal and magnetic fields:

$$
\begin{aligned}
E=0 ; & (\partial U / \partial S)_{V}=T+H,(\partial U / \partial V)_{S}= \\
& -\left(p_{\text {mech }}+p_{\text {magnetic }}\right)
\end{aligned}
$$


Eqs. (49)-(51) define the mechanical, magnetic pressure and electric pressure as defined in some literature of thermodynamics and electromagnetism [53].

Eq. (52) can be applied to study the thermoelectric effects as Joule, Thomson Seebeck, and Peltier effects. In this case, it is possible to predict the simultaneous influence of flow of electric current and heat on the properties of the system. Similarly, Eq. (53) can be applied to study the magnetocaloric effects [54].

Generally, this system of Equations solves many unsolved problems in physics [55].

\section{References}

[1] Callen, B., and Herbert B. 1985. Thermodynamics and an Introduction to Themostatistics. New York: John Wiley \& Sons.

[2] Fujimtu, M. 2007. Physics of Classical Electromagnetism. Springer.

[3] Serway, R. A., and Serway, J. W. 2010. Physics for Scientists and Engineers with Modern Physics. Eights edition, U.S.A.: Brooks Cole.

[4] Penfield, P., and Haus, H. A. 1967. Electrodynamics of Moving Media. Cambridge, Massachusetts: MIT Press.

[5] Chu, B. T. 1959. "Thermodynamics of Electrically Conducting Ftuids." Phys. Fluids 1 (5): 473-84.

[6] Tesla, N. 1919. "The Effect of Static on Wireless Transmission." Electrical Experimenter, 627-6587.

[7] Cannon, B., Hoburg, J., Stancil, D. and Goldstein, S. 2009. "Magnetic Resonant Coupling as a Potential Means for Wireless Power Transfer to Multiple Small Receivers." Power Electronics, IEEE Transactions 24 (7).

[8] Norton, J. 1987. "The Logical Inconsistency of the Old Quantum Theory of Black Body Radiation." Philosophy of Science 54 (3): 327-50.

[9] Trunov, G. M. 1983. "Correctness of the International System of Units in the Area of Electromagnetism." Journal of Measurement Techniques 26 (1): 9-16.

[10] Ketterle, W. 2002. "When Atoms Behave as Waves; Bose-Einstein Condensation and the Atom Laser." Reviews of Modern Physics 74 (10): 1131-51.

[11] Heikes, R. R., Roland, W., and Ure, W. J. 1961. Thermoelectricity: Science and Engineering. New York: Interscience Publishers Inc.

[12] Riffat, S. B., and Ma, X. 2003. "Thermoelectrics: A Review of Present and Potential Applications." Applied Thermal Engineering.

[13] Rowe, D. M. 2006. "Thermoelectrics Handbook: Macro to Nano." Taylor \& Francis Group.
[14] Van Herwaarden, A. W., and Sarro, P. M. 1986. "Thermal Sensors Based on the Seebeck Effect." Sensors and Actuators 10: 321-46.

[15] Weiling, L., and Shantung, T. U. 2004. "Recent Developments of Thermoelectric Power Generation." Chinese Science, Bulletin 49: 1212-9. http://dx.doi.org/10.1360/04we0037.

[16] Snyder, G. J., Fleurial, J. P., Caillat, T., Yang, R., and Chen, G. 2002. "Supercooling of Peltier Cooler Using a Current Pulse." Journal of Applied Physics 92: 1564-9.

[17] Buist, R. J. 1995. "The Extrinsic Thomson Effect." Proceedings of the 14th International Conference on Thermoelectrics, 27-30.

[18] Abdelhady, S. 2014. "An Entropy Approach to a Practical Limit of the Efficiency of Developed and Multi-juction Solar Cells.” J. Electromagnetic Analysis \& Applications 6: 383-90.

[19] Ryan, D. T. 2006. "Toward a Cognitive-Historical Understanding of Michael Faraday's Research: Editor's Introduction.” Perspectives on Science 14: 1-6.

[20] Sherwood, P. J., Henriksen, R. N., and Allen, J. R. 1969. “Approaches to Electromagnetic Induction.” Am. J. Phys. 377: 698-708.

[21] Stevens, C. F. 1965. The Six Core Theories of Modern Physics. Cambridge: MIT Press

[22] Abdelhady, S. 2013. "An Entropy Approach to Wireless Power Transmission by Magnetic Resonance." Applied Physics Research 5 (5): 29-35.

[23] Phan, M. H., and Yu, S. C. 2007. "Review of the Magnetocaloric Effect in Manganite Materials.” Journal of Magnetism and Magnetic Materials 308 (2): 325-40.

[24] Abdelhady, S., and Abdelhady, M. S. 2015. "An Entropy Approach to the Natures of the Electric Charge and Magnetic Flux." Journal of Electromagnetic Analysis \& Applications $\quad 7$ 265-75. http://dx.doi.org/10.4236/jemaa.2015.711028.

[25] Stevens, C. F. 1965. The Six Core Theories of Modern Physics. Cambridge: MIT Press.

[26] Tschoegl, N. 2000. Fundamentals of Equilibrium and Steady-State Thermodynamics. The Netherlands: Elsevier Science, Ltd: Amsterdam.

[27] Jackson, J. D. 1975. Classical Electrodynamics. New York: Wiley.

[28] Abdelhady, S. 2010. "A Fundamental Equation of Thermodynamics that Embraces Electrical and Magnetic Potentials." J. Electromagnetic Analysis \& Applications March, 162-9.

[29] Kutnjak, Z., and Rozic, B. 2013. "Indirect and Direct Measurements of the Electricalorific Effect." The Series of Engineering Materials 34: 147-82.

[30] Kolesnikova, V. V., Klinshpont, E. R., Kolikov, O. V., and Miinchuk, V. K. 1977. "The Para-magnetism of 
Modified Polyvinyl Chloride." Polymer Science 19: 1684-92.

[31] King Jr, W. H., Camilli, C. T., and Findeis, A. F. 1968. "Thin-Film Thermocouples for Differential Thermal Analysis.” Anal. Chem. 40 (8): 1330-5.

[32] Kumar, V., Singh, J., and Verma, S. S. 2009. "Performance Comparison of Some Common Thermocouples for Waste Heat Utilization." Asian Journal of Chemistry 21 (10): 62-5.

[33] Abdelhady, S. 2010. "An Approach to a Universal System of Units." Journal of Electromagnetic Analysis \& Applications 2: 549-56.

[34] Brevik, J. 1979. "Experiments in Phenomenological Electrodynamics and the Electromagnetic Energy-Momentum Tensor." Physics Reports 52 (3): 133-201.

[35] Hooyman, G. J., and De Groot, S. R. 1959. "Phenomenological Equations and Onsager Relations: The Case of Dependent Fluxes or Forces." Physica 21: 73-7.

[36] Feynman, R. P., Leighton, R. B., and Sands, M. 1963. "Lectures on Physics." Volume 1. Addison-Wesley, Reading MA.

[37] Laurendeau, N. M. 2005. "Statistical Thermodynamics." Cambridge University Press.

[38] Haaiday, D., Resnick, R., and Walker, J. 2004. Fundamentals of Physics. 7th Edition, New York: John Wiley \& Sons.

[39] Yunus, A. C., and Michael A. B. 2006. "Thermodynamics: An Engineering Approach." McGraw-Hill Science Engineering.

[40] Harman, T. C., and Honig, J. M. 1967. Thermoelectric and Thermomagnetic Effects and Applications. McGraw-Hill Book Company.

[41] Laurendeau, N. M. 2005. Statistical Thermodynamics. Cambridge University Press.

[42] Lebowitz, J. L. 1993. "Macroscopic Laws and Microscopic Dynamics, Time's Arrow and Boltzmann's Entropy.” Physica A 194: 1-97.
[43] Thomas, E. G., and Meadows, A. J. 1985. Maxwell's Equations and Their Applications. Bristol and Boston: Adam Hilger Ltd.

[44] Abdelhady, S. 2012. "A Thermodynamic Analysis of Energy Flow in Optical Fiber Communication Systems." Applied Physics Research 4: 22-9.

[45] Zahn, M. 1979. Electromagnetic Field Theory. New York: W'uey.

[46] Abdelhady, S. 2010. "Comments Concerning Measurements and Equations in Electromagnetism." $J$. Electromagnetic Analysis \& Applications 2: 677.

[47] Stephen, G. 2002. "Brush, Cautious Revolutionaries: Maxwell, Planck, Hubble.” Am. J. Phys. 70: 119. http://dx.doi.org/10.1119/1.1427310.

[48] Shockley, W., and Queisser, H. J. 1961. "Detailed Balance Limit of Efficiency of $p$-n Junction Solar Cells." Journal of Applied Physics 32: 510-9. http://dx.doi.org/10.1063/1.1736034.

[49] Abdelhady, S. 2013. "An Entropy Approach to Tesla's Discovery of Wireless Power Transmission." $J$. Electromagnetic Analysis \& Applications 5: 157-61.

[50] Kurs, A. 2007. "Power Transfer through Strongly Coupled Resonances." M.Sc. thesis, Massachusetts Institute of Technology.

[51] Mandip, J. S., and Hambleton, J. 2011. "Wireless Power Transmission Using Magnetic Resonance, Cornell College PHY312.” December 2011.

[52] Iordanishvili, E. K. 1981. "Thermodynamic Potential of Thermoelectricity" Journal of Thermoelectricity 41 (1).

[53] Lahoz, D. L. 1974. "Electromagnetic Forces.” University of Alberta, Edmonton, Alta, 1974

[54] Brevik, J. 1982. "Fluids in Electric and Magnetic Fields: Pressure Variation and Stability." Can. J. Phys. 60: 449.

[55] Abdelhady, S. 2017. "Innovative Solutions of Unsolved Problems and Misconceptions in Physics, International." Journal of Innovative Research in Science, Engineering $\begin{array}{llll}\text { and } & \text { Technology } & 6 & \text { (9). }\end{array}$ https://www.rroij.com/open-access/innovative-solutions-o f-unsolved-problems-and-misconceptions-in-physics-.pdf. 